\title{
Successful Pain Relief by Local Injection Under Ultrasound Guidance in a Patient with Anatomical Abnormality of Cervical 5 Nerve Root
}

\author{
Daqiang Zhao $\cdot$ Hong Zhang · Jian Chen · Tao Xu (D)
}

Received: May 8, 2020 / Published online: July 4, 2020

(c) The Author(s) 2020

Keywords: Anatomical abnormality; Cervical nerve root; Pain; Therapy; Ultrasound guidance

\section{Key Summary Points}

The incidence of nerve root anomalies is $14 \%$, the L5-S1 level being most commonly involved. As a result of agerelated changes, the exiting spinal nerves in the cervical spinal region are also frequently affected, or are approached because of cervical osteochondrosis or disc herniation management. Anomalies of the nerve roots often produce pain in the corresponding innervation. Sometimes, ultrasound is a valuable tool to depict an anatomical abnormality.

Digital Features To view digital features for this article go to https://doi.org/10.6084/m9.figshare.12452435.

D. Zhao $\cdot$ H. Zhang $\cdot$ T. Xu ( $ه)$

Department of Anesthesiology, Tongzhou People's

Hospital, Nantong 226300, China

e-mail: balor@sjtu.edu.cn

\section{Zhao}

Department of Anesthesiology, Jiahui International Hospital, Shanghai 200233, China

\section{J. Chen ( $\square)$}

Department of Orthopaedics, Tongzhou People's

Hospital, Nantong 226300, China

e-mail: tzcj@nttzhospital.com

The C5 nerve travels abnormally alongside the anterior edge of the anterior scalene muscle and may induce perception which distributes across the right backside of the neck and shoulder, with radiating pain to the right upper arm. Under ultrasound guidance, bolus injection of $2 \mathrm{ml} 0.15 \%$ ropivacaine plus Diprospan $2.5 \mathrm{mg}$ mixture to the C5 nerve root anterior to the anterior scalene muscle could relieve the shoulder and upper arm pain.

During the second visit, the patient complained that some back pain remains on the medial scapula area. Under ultrasound guidance, the dorsal scapular nerve was found to touch the posterior tubercle of the C6 transverse process. Bolus injection of $0.25 \%$ ropivacaine $2 \mathrm{ml}$ plus $10 \%$ glucose $3 \mathrm{ml}$ to the dorsal scapular nerve totally relieved the pain.

The C5 nerve bypasses the anterior edge of the scalenus muscle, and the dorsal scapular nerve may also be distributed abnormally and close to the posterior tubercle of the transverse process of C6. Frequent friction of the nerve causes pain in the medial part of the scapula.

T. $\mathrm{Xu}$

Department of Anesthesiology, Shanghai Jiao Tong University Affiliated Sixth People's Hospital, Shanghai 200233, China 


\section{CASE}

The incidence of nerve root anomalies is 14\% [1], the L5-S1 level being most commonly involved. As are result of age-related changes, the exiting spinal nerves in the cervical spinal region are also frequently affected, or are approached because of cervical osteochondrosis or disc herniation management. Anomalies of the nerve roots often produce pain in the corresponding innervation. Sometimes, ultrasound is a valuable tool to depict an anatomical abnormality [1].

The cervical 5 (C5) nerve travels abnormally alongside the anterior edge of the anterior scalene muscle and may induce perception which distributes across the right backside of the neck and shoulder, with radiating pain to the right upper arm (Fig. 1a-c). Under ultrasound guidance (Fig. 1b), bolus injection of $2 \mathrm{ml} 0.15 \%$ ropivacaine plus betamethasone $2.5 \mathrm{mg}$ mixture to the C5 nerve root anterior to the anterior scalene muscle could relieve the shoulder and upper arm pain. During the second visit, the patient complained some back pain remains on the medial scapula area.

Under ultrasound guidance, the dorsal scapular nerve was found to touch the posterior tubercle of the C6 transverse process (Fig. 1d). Bolus injection of $0.25 \%$ ropivacaine $2 \mathrm{ml}$ plus $10 \%$ glucose $3 \mathrm{ml}$ to the dorsal scapular nerve totally relieved the pain. The C5 nerve bypasses the anterior edge of the scalenus muscle, and the dorsal scapular nerve may also be distributed abnormally and close to the posterior tubercle of the transverse process of C6. Frequent friction of the nerve causes pain in the medial part of the scapula. The patient provided their informed consent to publish the article and all procedures were conducted as part of standard care/treatment. 

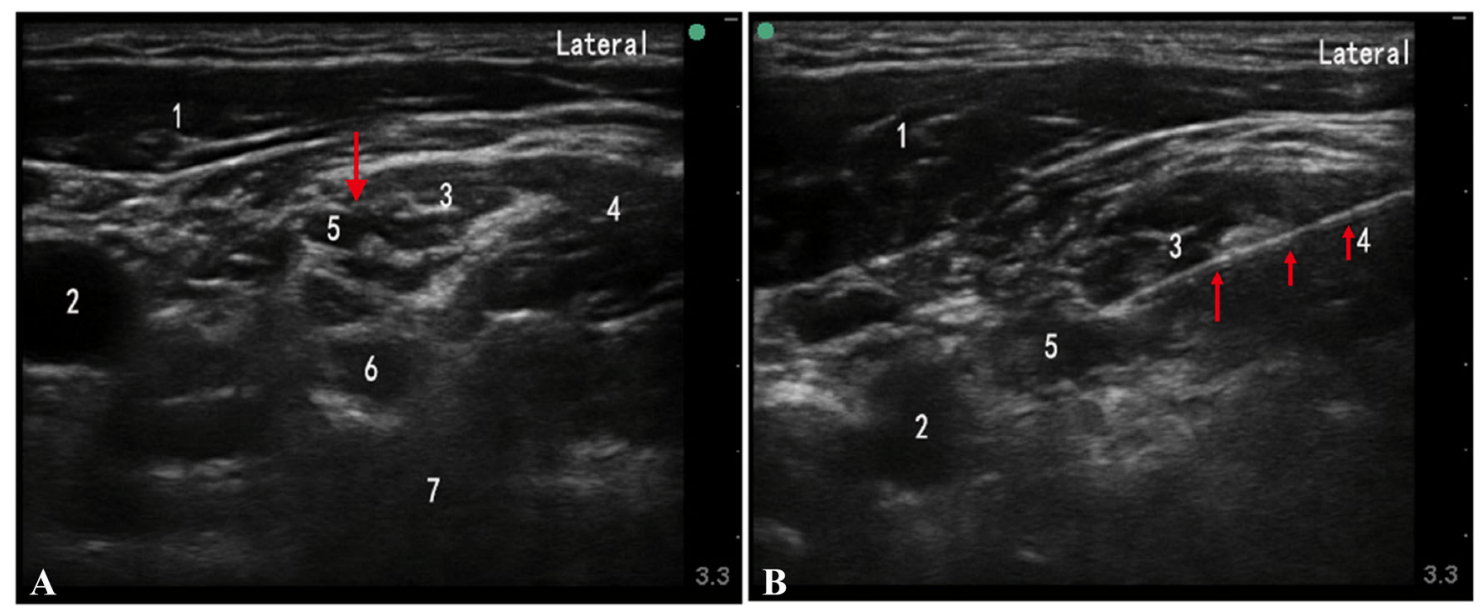

1.Sternocleidomastoid muscle; 2.Arteria carotis communis; 3. Anterior scalenus muscle; 4. Scalenus medius; 5. C5 nerve; 6. C6 nerve root; 7. Transverse process of $\mathrm{C} 6$

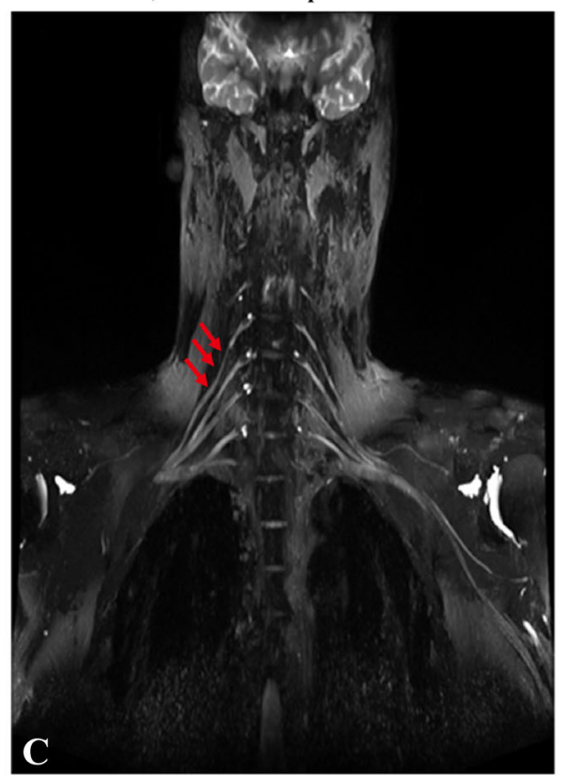

Fig. 1 a Under ultrasound scanning, the C5 nerve (red arrow) travels abnormally alongside the anterior edge of the anterior scalene muscle, but not in the intermuscular sulcus. b Under ultrasound guidance, a needle (red arrows) punctured the C5 nerve root anterior to the anterior scalene muscle. c MRI of the brachial plexus also

\section{ACKNOWLEDGEMENTS}

We thank the participants of the study.

Funding. No funding or sponsorship was received for this study or publication of this article. Tongzhou People's Hospital funded the journal's Rapid Service Fee.

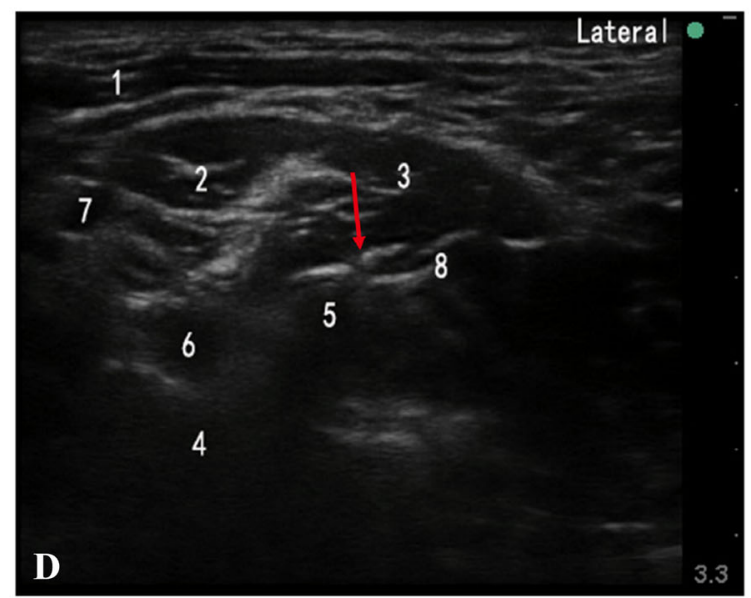

1.Sternocleidomastoid muscle; 2.Arteria carotis communis; 3. Anterior scalenus muscle; 4. Transverse process of $\mathrm{C6} ; 5$. Posterior tubercle of transverse process of C6; 6. C6 nerve root; 7. C5 nerve; 8. Dorsal scapular nerve

confirmed that the part of the C5 nerve away from the transverse process moved forward. d The dorsal scapular nerve was found to touch the posterior tubercle of the $\mathrm{C} 6$ transverse process; the nerve showed a little bit of edema. Under ultrasound guidance, a needle punctured the dorsal scapular nerve

Authorship. All named authors meet the International Committee of Medical Journal Editors (ICMJE) criteria for authorship for this article, take responsibility for the integrity of the work as a whole, and have given their approval for this version to be published. Daqiang Zhao and Hong Zhang contributed equally to the manuscript. 
Disclosures. Daqiang Zhao, Hong Zhang, Jian Chen, and Tao $\mathrm{Xu}$ have nothing to disclose.

Compliance with Ethics Guidelines. The patient provided their informed consent to publish the article and all procedures were conducted as part of standard care/treatment.

Open Access. This article is licensed under a Creative Commons Attribution-NonCommercial 4.0 International License, which permits any non-commercial use, sharing, adaptation, distribution and reproduction in any medium or format, as long as you give appropriate credit to the original author(s) and the source, provide a link to the Creative Commons licence, and indicate if changes were made. The images or other third party material in this article are included in the article's Creative Commons licence, unless indicated otherwise in a credit line to the material. If material is not included in the article's Creative Commons licence and your intended use is not permitted by statutory regulation or exceeds the permitted use, you will need to obtain permission directly from the copyright holder. To view a copy of this licence, visit http:// creativecommons.org/licenses/by-nc/4.0/.

\section{REFERENCE}

1. Kadish LJ, Simmons EH. Anomalies of the lumbosacral nerve roots. An anatomical investigation and myelographic study. J Bone Joint Surg Br. 1984;66:411-6.

2. Coraci D, Cruciani A, Giovannini S, Bernetti A, Santilli V, Padua L. Ultrasound to depict anatomical abnormality: an example of potential alliance of rehabilitation professionals. Med Ultrason. 2018;1: 117-8. 